\title{
Synchrotron FTIR Micro-spectroscopy Studies Demonstrate the Release of Ceramide Molecules from a Ceramide-containing UV-curable Acrylic Adhesive Resin
}

\author{
Hiroshi Takahashi ${ }^{1 *}$, Ryota Watanabe ${ }^{2}$, Kenichi Nishimura ${ }^{2}$, and Taro Moriwaki ${ }^{3}$ \\ ${ }^{1}$ Biophysics Laboratory, Division of Pure and Applied Science, Graduate School of Science and Technology, Gunma University, 4-2 Aramaki, \\ Maebashi, Gunma 371-8510, JAPAN \\ ${ }^{2}$ Medical Engineering Laboratory, ALCARE Co., Ltd. 1-21-10 Kyojima, Sumida-ku Tokyo 130-0046 JAPAN \\ ${ }^{3}$ Japan Synchrotron Radiation Research Institute (JASRI/Spring-8), 1-1-1 Kouto, Sayo, Hyogo 679-5198, JAPAN
}

\begin{abstract}
A recent study has shown that the addition of ceramide to adhesive materials of medical sheets or tapes improves or maintains skin barrier functions despite the irritation and damage caused by the repeated removal of the sheet or tape. This fact may imply that ceramide molecules are released from the adhesive material and penetrate the skin. In this study, we investigated whether ceramide molecules are released from a UV-curable acrylic adhesive resin $\left(\operatorname{acResin}^{\circledR}\right)$ sheet containing ceramide molecules attached to a cultured skin sample by estimating the local ceramide concentration from the data obtained by synchrotron Fourier transform infrared micro-spectroscopy analysis. Since section samples of uniform thickness could not be prepared, the intensity data of the amide I peak originating from ceramide were normalized by using the intensity data of the ester peak originating from the resin matrix. This analysis enabled the quantification of the change in local ceramide concentrations in the sheet samples. This result indicates that ceramide molecules were released from the $\operatorname{acResin}^{\circledR}$ sheet attached to the cultured skin sheet.
\end{abstract}

Key words: ceramide, release, adhesive resin, medical sheet, cultured skin

\section{INTRODUCTION}

Medical adhesive tapes or sheets are widely used for many purposes, for example, protecting wounds, fixing various medical instruments, etc ${ }^{1)}$. Repeated use of these tapes or sheets, i.e., recycling of application and removal, possibly could cause skin damage ${ }^{2,3}$. This skin damage is due to the removal of the epidermal layer of the skin. This problem is serious for newborn babies, because their epidermal layer has not grown sufficiently, and for elderly people with weak skin function ${ }^{4,5)}$.

One of the functions of the epidermal layer is to act a barrier to protect the body against external stimuli, microbial pathogens, chemical and physical stresses, etc. The function is mainly carried out by the stratum corneum, which is the outermost layer of the skin ${ }^{6}$. At the molecular level, ceramide is the most abundant component of the intercellular lipids in the stratum corneum ${ }^{7)}$. Several of studies have shown that a lamellar structure formed mainly by ceramide molecules is an indispensable key structure for the barrier function of the skin ${ }^{6-8}$. The lack of ceramide has been also reported to cause skin barrier dysfunction $^{6,9-12)}$.

The above fact naturally leads to the idea that by adding ceramide to the adhesive material of the medical tapes or sheets, it is possible to create a new, skin-friendly medical tape or sheet. Numata et al. ${ }^{13)}$ investigated the effectiveness of this idea by performing a patch test for healthy individuals and ostomates. In their study, ceramides were added to the hydrocolloid adhesive materials of the faceplate of the stoma appliance model used in the patch test. The study showed that the addition of ceramide contributes towards improving or maintaining the skin barrier function, judging from the transepidermal water loss (TEWL) values.

Acrylic adhesive resins are widely used for various medical sheets and tapes ${ }^{14)}$. Recently, we ${ }^{15)}$ have found that

\footnotetext{
* Correspondence to: Hiroshi Takahashi, Biophysics Laboratory, Division of Pure and Applied Science, Graduate School of Science and Technology, Gunma University, 4-2 Aramaki, Maebashi, Gunma 371-8510, JAPAN

E-mail: hirotakahashi@gunma-u.ac.jp

Accepted December 4, 2018 (received for review September 13, 2018)

Journal of Oleo Science ISSN 1345-8957 print / ISSN 1347-3352 online

http://www.jstage.jst.go.jp/browse/jos/ http://mc.manusriptcentral.com/jjocs
} 
in a UV-curable acrylic adhesive resin, acResin ${ }^{\otimes 16,17)}$, ceramide molecules exhibit a thermal-dependent phase behavior that is similar, but not identical to that exhibited by a pure ceramide sample ${ }^{18)}$, and that the ceramide molecules in acResin ${ }^{\circledast}$ can also form a lamellar structure similar to an intercellular lamellar structure in the stratum corneum. There are two possible explanations for the above-mentioned skin-barrier function-improvement effect ${ }^{13)}$ of the addition of ceramide to the adhesive material. One possibility is that the sheet containing the lamellar structures (formed by ceramide molecules) itself functions as a barrier on the surface of the skin. The other possibility is that ceramide molecules released from the adhesive materials penetrate the stratum corneum region of the skin. As a result, the skin barrier function is maintained and improved by compensating for the original number of ceramide molecules in the stratum corneum region which was decreased due to the skin irritation. Of course, it might be possible that both mechanisms work together.

The aim of this study was to investigate whether ceramide molecules are released from adhesive resin materials containing ceramide molecules. To this end, we performed Fourier transform infrared micro-spectroscopy (FTIRM) measurements on the section samples of a model medical sheet made from the acResin ${ }^{\circledast}$ sheet contained ceramide molecules and was attached to a cultured skin sheet. If ceramide molecules are released from the ceramide-containing resin and transferred into the cultured skin sheet, the ceramide concentrations of the adhesive sheet should decrease near the attached region. FTIRM measurements using small infrared beams of the micrometer order provide information on the chemical distribution of materials with spatial resolution in the micrometer scale. A small but strong IR beam is needed to obtain high-quality FTIRM data. In this study, we used an infrared synchrotron beam from the storage ring at the SPring-8 synchrotron radiation facility (Hyogo, Japan). This short note reports that ceramide molecules are released from the acResin ${ }^{\circledR}$ sheet attached to a cultured skin sheet.

\section{EXPERIMENTAL PROCEDURES}

\subsection{Materials}

In this study, we used synthetic ceramide molecules. The synthetic D-erythro-ceramide, $(2 S, 3 R)$-2-octadecanoylamino-octadecane-1,3-diol was obtained from Takasago International Corp. (Tokyo, Japan), which has both a chemical and optical purity of more than $95 \%$ quality, according to the supplier's information. The synthesis method of the ceramide has been reported by Ishida $e t a l .{ }^{19)}$ The use of $\operatorname{acResin}^{\circledR}$ as a UV-curable acrylic adhesive resin material was the same as that in our previous study ${ }^{15}$. The prepolymers with a mean molecular weight of $\sim 200,000$ were sup- plied by BASF (Ludwigshafen am Rhein, Germany). According to the BASF's product catalogue, the acResin ${ }^{\circledR}$ is made from benzophenone groups and the polymer of butyl acrylate. Polyisobutylene (Himol, grate $5.5 \mathrm{H}$ ) with an average molecular weight of 53,000 was obtained from JXTG Nippon Oil \& Energy Corporation(Tokyo, Japan). Styrene isoprene thermoplastic elastomer (SIS5229) was obtained from JSR Corporation(Tokyo, Japan). Liquid paraffin (MORESCO WHITE P-350P) with a kinematic viscosity of $67.65 \mathrm{~mm}^{2} / \mathrm{s}$ at $40^{\circ} \mathrm{C}$ was obtained from MORESCO Corporation(Tokyo, Japan). As carboxymethylcellulose (CMC), SUNROSE ${ }^{\circledR}$ MAC800LC of Nippon Paper Industries Co., Ltd. (Osaka, Japan) was used. SUNROSE ${ }^{\circledR}$ MAC800LC is a sodium CMC with a OD1\% viscosity of 6,000 - 10,000. Reconstructed human skin tissue culture models (EpiSkin ${ }^{\mathrm{TM}}$ ) with a size of $0.38 \mathrm{~cm}^{2}$ were obtained from Nikoderm Research Inc. (Osaka, Japan). The EpiSkin ${ }^{\mathrm{TM}}$ model skin was developed by E. Tionis et $a{ }^{20}{ }^{20}$; it consists of a bovine collagen matrix surface with a film of human collagen, on which epidermal cultures obtained from human keratinocytes are placed. The EpiSkin ${ }^{\mathrm{TM}}$ model skin has been widely used for phototoxicity testing, chemical irritancy testing, etc. ${ }^{21}$ The EpiSkin ${ }^{\mathrm{TM}}$ cultured skin was fixed to a plastic cup as shown in Fig. 1 (a), which was placed in 12-well culture dishes.

\subsection{Preparation of sheet samples}

The preparation method of the UV-curable acrylic adhesive resin containing ceramide molecules has been reported previously ${ }^{15}$. Only a brief description of this method has been provided here. To mix the ceramide powder and the prepolymers of acResin ${ }^{\circledR}$ uniformly, both the ceramide powder sample and the viscous fluid of the prepolymer were heated above the melting temperature of pure ceramide. The hot-mixed samples were dropped onto a hot plate at $125^{\circ} \mathrm{C}$ and were spread into sheets with a thickness of $\sim 200 \mu \mathrm{m}$. The sheet samples were kept at $80^{\circ} \mathrm{C}$, during which they were simultaneously irradiated with UV light to cure the materials. After the irradiation using UV light, the acResin $^{\circledast}$ sheets were kept at room temperature for five days. Samples with two different ceramide concentrations were prepared. One sample contained 2 wt $\%$ ceramide and the other contained $9 \mathrm{wt} \%$ ceramide.

As a control, ceramide-containing acResin ${ }^{\circledast}$ sheets that were not attached to cultured skin sheet must be measured. The sheets were, however, extremely soft and sticky; hence it was difficult to prepare section samples with a constant thickness. In this study, to add hardness to the sheet samples, the acResin ${ }^{\circledR}$ sheet was fixed to a relatively hard rubber sheet, and then it was cut to prepare a section sample. The rubber sheets were prepared as follows. First, the styrene isoprene thermoplastic elastomer (SIS5229) and liquid paraffin (MORESCO WHITE P-350P) were mixed at $200^{\circ} \mathrm{C}$ by using a kneader and then the 
(a)

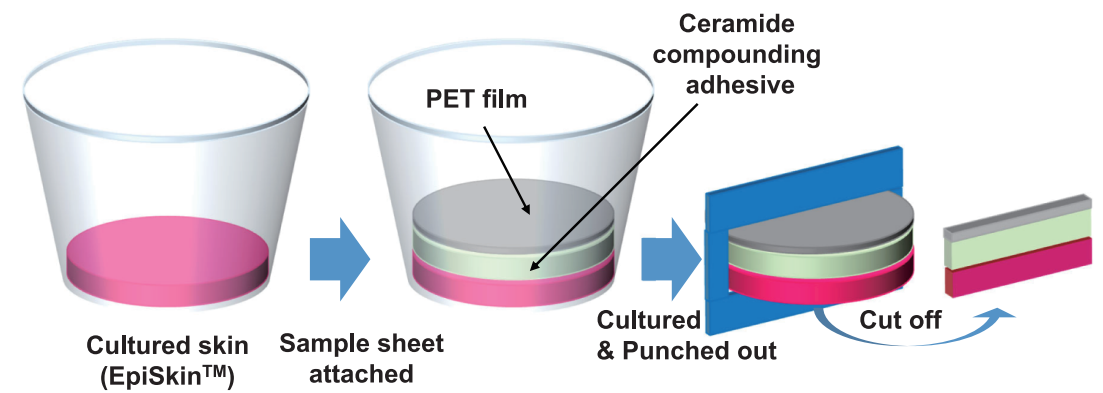

Scan direction

(b)

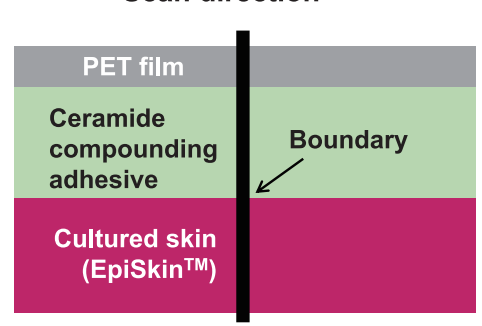

Fig. 1 (a) Schematic diagram on the EpiSkin ${ }^{\mathrm{TM}}$ sample and the preparation of the section samples (see text). (b) Schematic representation on the scan direction of the samples (see text).

mixture was cooled to about $25^{\circ} \mathrm{C}$. Polyisobutylene and

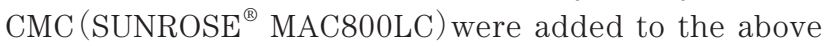
mixture, followed by stirring with a kneader to obtain a homogeneous mixture. The composition of the mixture was $40 \mathrm{wt} \%$ of polyisobutylene, $20 \mathrm{wt} \%$ of styrene isoprene thermoplastic elastomer and fluid paraffin, and $40 \mathrm{wt} \%$ of CMC. Finally, the mixture was rolled to obtain a rubber sheet with a thickness of $\sim 200 \mu \mathrm{m}$. Hence, we will call it PIB rubber. In order to facilitate the handling of the adhesive sheet prepared as described above, a 50- $\mu \mathrm{m}$ thick polyethylene terephthalate (PET) film treated on one side silicone was attached to one side of the adhesive sheet (see Fig. 1(a)). The PET films $($ PET50 $\times 1$ A3) were obtained from Nippa Corporation (Suita, Osaka, Japan).

\subsection{Preparation of the section samples}

Before preparing section samples, the acResin ${ }^{\circledR}$ sheet samples attached to cultured skin were sealed in a plastic box, which was supplied by the manufacturer of the EpiSkin ${ }^{\text {TM }}$ model skin as a culture kit, for one day or three days. During these periods, the culture media provided by the manufacturer were replaced at 24-hours intervals, and the temperature was kept at $37^{\circ} \mathrm{C}$. The manufacturer recommends maintaining the $\mathrm{CO}_{2}$ concentration at $5 \%$ as a suitable culture condition. To achieve this, a bag of CULTUREPAL $^{\circledR}$ (Mitsubishi Gas Chemical Company, Inc., Tokyo), which produced $5 \% \mathrm{CO}_{2}$, was put in the plastic box. Before cutting, the acResin ${ }^{\circledR}$ sheet samples attached to the PIB rubber sheet were kept at room temperature for three days.

The adhesive resin sheet samples adhering to the cultured skin or PIB rubber sheet were cut out to make section samples by using a Tissue-Tek Cryo3 microtome/ cryostat (Sakura Finetek USA, Inc., Torrance, CA). The Tissue-Teck O.C.T. (Sakura Finetek USA, Inc., Torrance, $\mathrm{CA}$ ) compound was used as the embedding agent. Since the acResin $^{\circledR}$ sheet samples are extremely soft and sticky at a room temperature, the samples were cooled down to $75^{\circ} \mathrm{C}$ by using liquid nitrogen and then, the cutting operation was carried out by using the lowest temperature setting of the microtome chamber $\left(-35^{\circ} \mathrm{C}\right)$. The typical time duration from the preparation of the section samples to the FTIRM measurements at the SPring- 8 facility was seven days. During this period, the samples attached to cultured skin sheets were stored at $5^{\circ} \mathrm{C}$ while those attached to PIB rubber sheets were stored at room temperature.

\subsection{Synchrotron Fourier transform infrared micro -spec- troscopy (FTIRM) measurements}

FTIRM measurements using synchrotron radiation were performed at the infrared-microscope station beamline BL43 IR of the SPring-8 facility (Hyogo, Japan) ${ }^{22,23)}$. In the BL43IR, IR absorption spectra from a narrow region of the order of several microns can be obtained with a system combining a Bruker Vertex 70 spectrophotometer and Hyperion 2000 infrared microscope. The section samples were measured by placing them on $\mathrm{CaF}_{2}$ plate. In this experiment, the spectral data were recorded using an aperture of $10 \mu \mathrm{m} \times 10 \mu \mathrm{m}$ with $8 \mu \mathrm{m}$ steps in a one-dimensional direction. The scanning was performed along the normal direction of the sheets (see Fig. 1(b)). IR spectra were obtained in the wave number range of $600-4000 \mathrm{~cm}^{-1}$ with a nominal resolution of $4 \mathrm{~cm}^{-1}$, by averaging 128 signal data. 


\subsection{Conventional Fourier transform infrared spectroscopy (FTIR) measurements}

Conventional FTIR measurements were performed by using an FTIR-8300 Spectrophotometer (Shimadzu Co., Kyoto, Japan). The spectrophotometer can measure in both the conventional transmission mode and attenuated total reflection (ATR) mode. Sheet samples were measured mainly using the ATR mode, while the powder samples of raw materials (ceramide, etc.) were measured using the transmission mode.

\subsection{Three-dimensional (3D) laser microscopic observation}

A preliminary evaluation of the thickness of the sliced sample was carried out using a Lasertec OPTELICS H 1200 three-dimensional (3D) laser microscope (Lasertec Corporation). In order to achieve the same conditions as those for the FTIRM measurements, the sample was placed on the $\mathrm{CaF}_{2}$ plate and measured with an observation magnification of $50 \times$. In the thickness measurements, based on the microscopic images of the shape the samples, we intended to evaluate the same position as that measured by FTMIR technique as much as possible.

\section{RESULTS AND ANALYSIS}

\subsection{D microscopic observation of the section samples}

Before performing the FTIRM measurements, we carried out 3D microscopic observations to obtain a rough estimate of the thickness of the section samples. Figure 2 shows an example of the thickness profile data and the observed microscopic images. The sectioned sheet sample in Fig. 2 was prepared after subjecting to attach human skin. It can be seen that the thickness of the samples is not constant and varies depending on the location. For this sample, the mean thickness was $\sim 26 \mu \mathrm{m}$ and the standard deviation was $\sim 9 \mu \mathrm{m}$, although the intended thickness of the prepared was of $20 \mu \mathrm{m}$. The maximum height was $39 \mu \mathrm{m}$ and the minimum height was $5 \mu \mathrm{m}$ in the region of $650-1050$ $\mu \mathrm{m}$. The thicknesses of other samples attached cultured skin were also not uniform and tended to vary by 20 to $50 \%$. The samples were cut in a low-temperature environment, but since the acResin ${ }^{\circledR}$ sheet samples were flexible and sticky, at present, it is impossible to prepare section samples with a uniform thickness. As described below, in this study, we analyzed the FTIRM data taking the non-uniformity of the thickness of the samples into consideration.

\subsection{FTIRM spectroscopy}

In this study, from the change of ceramide concentrations of the acResin ${ }^{\circledR}$ sheet samples attached to a cultured skin, we judged whether ceramide molecules were released from the adhesive resin containing ceramide molecules. One of the reasons for using cultured skins is that the re- (a)

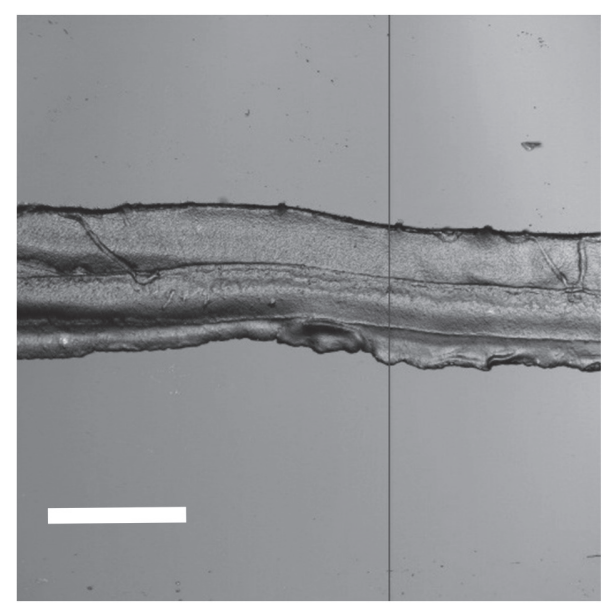

(b)

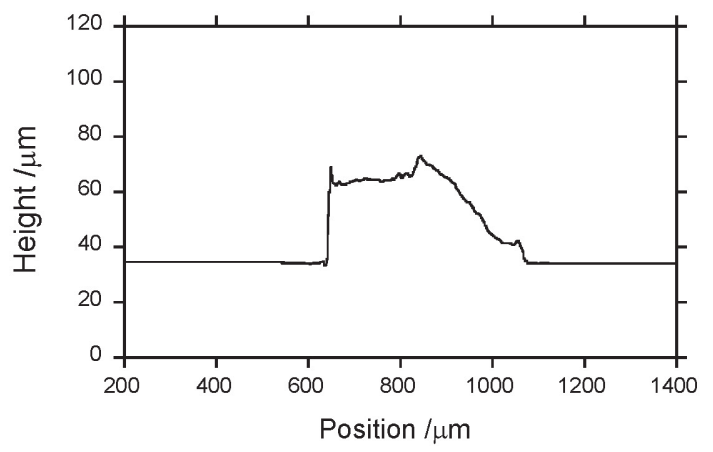

Fig. 2 (a) Microscopic image of a section sample of $\operatorname{acResin}^{\circledR}$ sheet. After attaching to human skin for three days, the sample was peeled from the skin and then cut off. Several thin stratum corneum layers attached the bottom of the sheet. The scalar bar is $400 \mu \mathrm{m}$. (b) Plot of the estimated thickness by the 3D laser microscope at the line position indicated by the vertical line in Fig. 2 (a).

producibility of the results is expected to be higher than that obtained by using real human skins. The period of the attachment of the resin sheets to the skin was one to three days. Although differences in the patterns of the subjects' daily lives during this period may affect the results, it is difficult to control the patterns of the subjects' daily lives in detail. Hence, we decided to use cultured skin in this study. To determine the ceramide concentrations, we used the intensities of the so-called amide I band observed at $1600-1650 \mathrm{~cm}^{-1}$ derived from the amide bond of the ceramide molecule. The amide $\mathrm{I}$ band is due to the $\mathrm{C}=\mathrm{O}$ stretching vibrations of amide bonds. Figure $3(\mathrm{a})$ shows a typical IR spectrogram of an acResin ${ }^{\circledR}$ resin sheet sample containing 2 wt\% ceramide obtained by FTIRM measurements. The acResin ${ }^{\circledR}$ resin is made of an acrylic polymer with benzophenone groups, and has no amide bonds ${ }^{16,17)}$. 

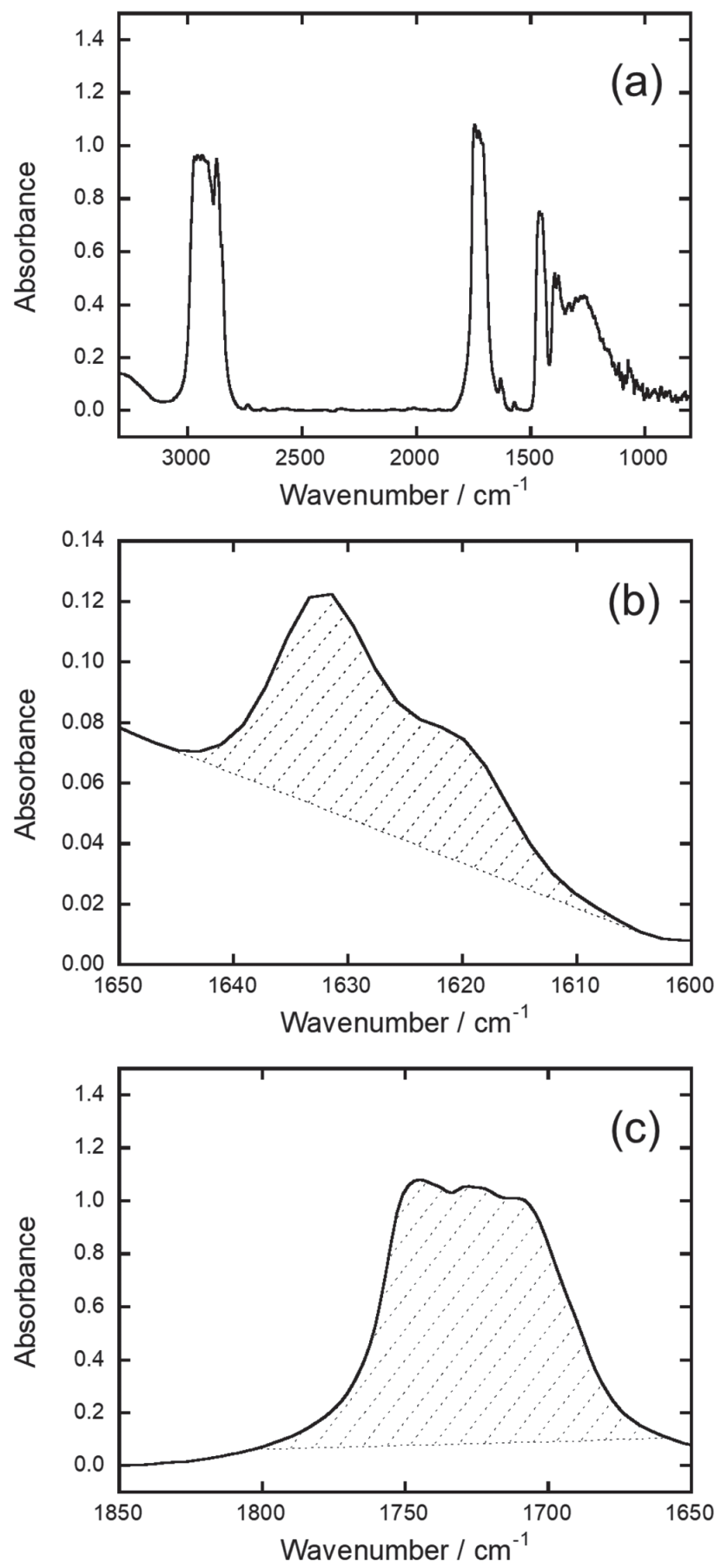

Fig. 3 (a) Infrared absorbance spectrum of ceramidecontaining acResin ${ }^{\circledR}$ sheet sample attached to cultured skin for three days obtained by synchrotron FTIRM measurements. (b) Expansion plot of (a) near the amide I peak. This figure explains the procedure of the integration of the amide I peak. See body text. (c) Expansion plot of (a) near the ester peak. This figure explains the procedure of the integration of the ester peak. See body text.
In fact, by using a conventional FT-IR spectrometer (data not shown). we confirmed that an acResin ${ }^{\circledR}$ sheet sample containing no ceramide give no distinct peak at 1500 $1700 \mathrm{~cm}^{-1}$. The intensities of the amide I peak were obtained by integrating the area surrounded by a straight background line within the spectra, as shown in Fig. 3(b).

The above-mentioned 3D microscopic measurements revealed that the thicknesses of the sheet samples are not uniform but depended on the positions. Thereby, one cannot directly determine the ceramide concentration of the observed position from the intensity of amide I band. It is necessary to compensate for the variation in thickness. Here, we estimated the thickness of the sheet samples from the intensities of the peak observed at around 1660$1800 \mathrm{~cm}^{-1}$ (Fig. 3(c)). The peak corresponds to the $\mathrm{C}=\mathrm{O}$ stretching vibrations of ester bonds. This peak does not appear in the IR spectra of pure ceramide powder samples. Hence, it is possible to normalize the intensities of the amide I originating from the ceramide molecules by dividing by the ester peak intensities, Thus, from the FTIRM data, one can estimate the ceramide concentration at each observed position, although the thickness of each observed position is different. From the comparison with the data obtained by conventional FTIR measurements (data not shown), the peak observed at around $1660-1800 \mathrm{~cm}^{-1}$ in the present FTIRM spectrum(Fig. 3(c)) seemed to be only slightly saturated. This is because the present experiment conditions (the thickness of the samples) were set so that a weak amide I peak could be clearly detected. However, for the scanlines at approximately the same position judged from the microscopic images of the shape the samples, the thickness distribution obtained from 3D laser microscopy was the same as that estimated by the FTIRM data. Based on these results, we assumed that the slight saturation of the peak observed at around $1660-1800 \mathrm{~cm}^{-1}$ can be considered negligible.

We recorded FTIRM data with three different lines for each sample along the normal direction of the sheet plane. The scan lines were $\sim 200-300 \mu \mathrm{m}$ apart from each other. To compare and average these three data obtained from different positions of the same sample, the boundary between the acResin ${ }^{\circledR}$ sheet and the cultured skin or the PIB rubber sheet had to be determined. The chemical compositions of acResin ${ }^{\circledR}$ containing ceramide molecules entirely differ from those of a cultured skin or a PIB rubber sheet. In other words, these materials yield different IR spectra. We could easily determine the boundary from the change of IR spectra as shown in Fig. 4. The determined boundary position was set to the origin i.e., 0 in Fig. 5 .

Figure 5 shows the plots of normalized amid I peak intensities, i.e., the values obtained by dividing the observed amide I peak intensity by the observed ester peak intensity, as a function of the position from the boundary. These graphs can be interpreted as the change of the ceramide 


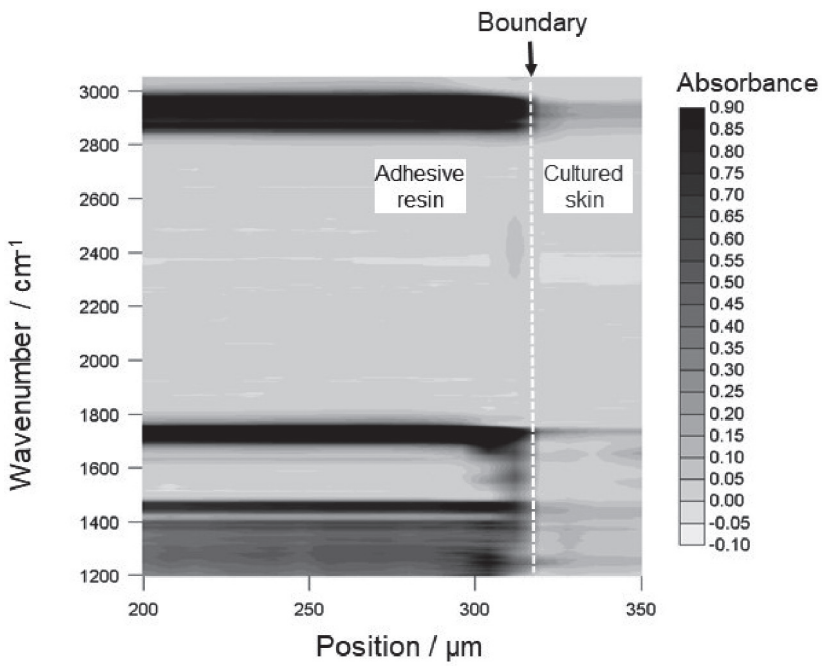

Fig. 4 Two-dimensional grey plot to present the changing behavior of infrared absorbance spectra depending on the observed positions obtained by synchrotron FTIRM measurements. The spectra data are for ceramide-containing acResin ${ }^{\circledR}$ sheet sample attached to cultured skin for three days.

concentrations as a function of the position. For the sample attached to PIB rubber sheets (Fig. 5(a)), as expected, there was no change of the ceramide concentration depending on the position. This result also indicated that the distribution of ceramide molecules was homogeneous in the $\operatorname{acResin}^{\circledR}$ sheet sample.

On the other hand, in case of acResin ${ }^{\circledR}$ sheets attached to the cultured skin sheets, a significant decrease of the ceramide concentrations was observed in the terminal region directly attached to the cultured skin (Fig. 5(b) and (c)). The decrease of ceramide concentration for the resin sample attached to the cultured skin for three days was greater than that for the resin sample attached to the cultured skin for only one day. From the results shown in Fig. 5(b) and (c), we concluded that when the ceramide-containing acResin ${ }^{\circledR}$ sheet samples are attached to cultured skin sheets, the ceramide molecules are released from the sheets.

Ceramide molecules originally exist in the cultured skin regions. For the method used in this study, it is impossible to distinguish between the native ceramide molecules of the cultured skin sheets and the ceramide molecules originating from the acResin ${ }^{\circledR}$ sheet. For these reasons, at present, we cannot determine whether the ceramide molecules released from the ceramide-containing acResin ${ }^{\circledR}$ sheet samples penetrate the cultured skin.

\section{DISCUSSION}

In the present study, we demonstrated the release of ce-

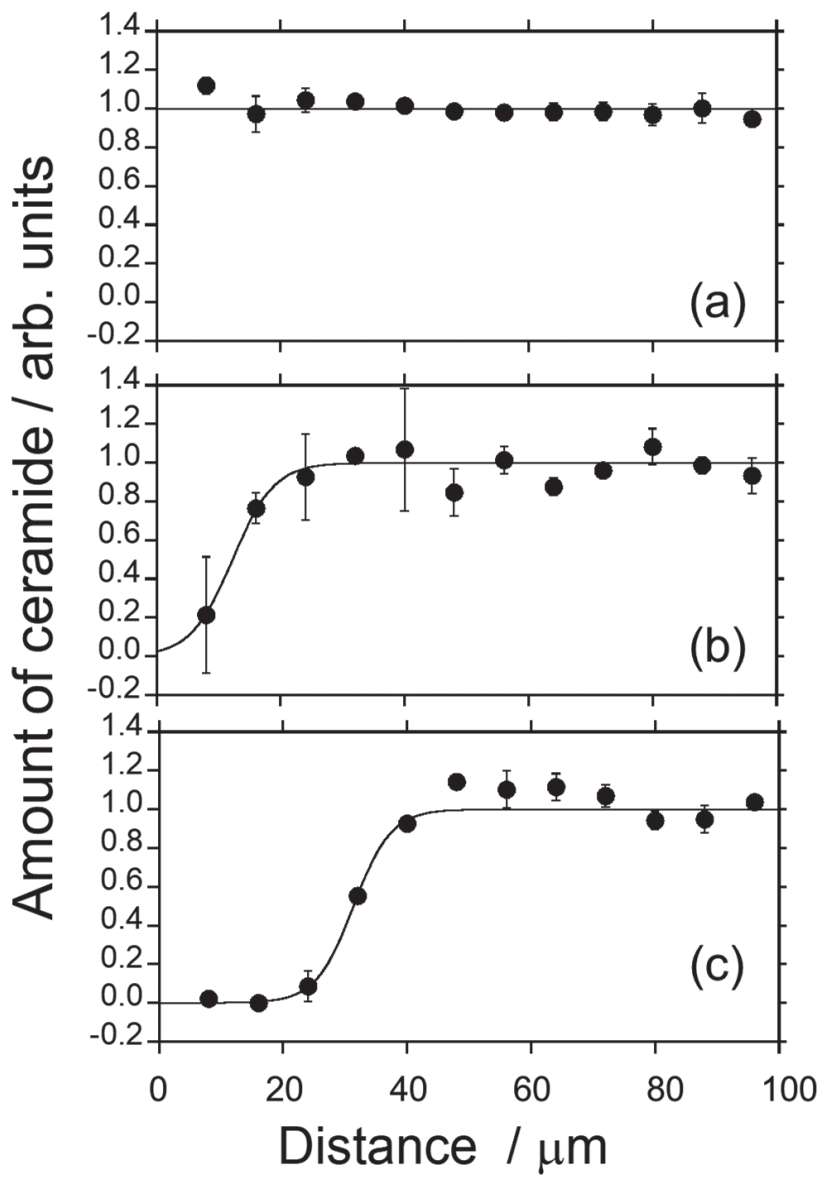

Fig. 5 Plots of the amount of ceramide (ceramide concentrations) estimated from the absorbance data of synchrotron FTIRM measurements as a function of distance from the interface between the $\operatorname{acResin}^{\circledR}$ sheet and cultured skin or rubber sheet. (a) acResin $^{\circledR}$ sheet attached to a PIB rubber sheet, (b) acResin ${ }^{\circledR}$ sheet attached to a cultured skin sheet for one day before cutting to a section, and (c) for three days. The error bars indicate the standard deviation calculated from the three data. The lines are guides for the eyes.

ramide molecules from the UV-curable acrylic adhesive resin $\left(\operatorname{acResin}^{\circledR}\right)$ sheets attached to cultured skin sheets from the results obtained by FTIRM spectroscopy. However, we cannot distinguish whether the ceramide molecules found in the cultured skin sheets were from the ceramide-containing acResin ${ }^{\circledR}$ sheets or were those existing originally in the stratum corneum of the cultured skins. Although strictly, we have no experimental evidence that the released ceramide molecules penetrate into the cultured skin sheet, it is natural to interpret that the released ceramide penetrated the cultured skin. This is because there is no other place for the ceramide molecules to migrate to. 


\section{Release of Ceramide Molecules from a Ceramide-containing Adhesive Resin}

Recently, in our previous study, using X-ray diffraction measurements, we have shown that when melted ceramide molecules in the acResin ${ }^{\circledR}$ sheet are cooled to room temperature, the ceramide molecular assembly forms a lamellar structure in which the hydrocarbon chains of the ceramide are packed into a hexagonal lattice. However, the corresponding diffraction peaks were relatively broad, suggesting that the ceramide molecules were packed into a somewhat disordered lattice. The structure of this lattice different from that of the pure ceramide molecule crystals, but the ceramide molecules must be in a solid state. It is likely that ceramide molecules in a solid state cannot diffuse; even if this is possible, the molecules diffuse extremely slowly in the acResin ${ }^{\circledR}$ sheet sample. Thus, we hypothesized that oily substances may have seeped out from the cultured skin, thereby solubilizing the ceramide molecules in the acResin ${ }^{\circledR}$ sheet, and allowing them to penetrate the cultured skin sheets. We would like to explore this hypothesis in future studies.

Although we also prepared the resin samples attached to human skin as shown in Fig. 1, we could not obtain enough quality data to help us draw a clear conclusion. This was due to the extreme difficulty associated with creating the section samples. It is possible to cut the sheet samples together with the cultured skin. On the other hand, it is not easy to cut the sheet sample together with human skin. To do this, the skin, including the dermis must be cut out surgically. Conducting this surgical treatment poses an ethical problem. When the acResin ${ }^{\circledR}$ sheet was peeled off from the skin, only a thin stratum corneum remained on the surface of the sheet sample. Hence, the mechanical strength of this sample is lower than that of the sample attached to cultured skin. As a result, it was more difficult to prepare the sectioned samples. However, our preliminary analysis of the data obtained from the resin samples attached to human skin suggest that ceramide molecules might be released from the acResin ${ }^{\circledR}$ sheet.

The present results suggest that only ceramide molecules that exist at a narrow region near the interface of the cultured skin and sheet penetrate the skin during the attachment period of several days (Fig. 4(b) and (c)). The sheets or tapes for fixing ostomy appliances are usually replaced over a period of several days. For such applications, preparing sheets and tapes containing ceramide molecules only near the thin adhesion surface may be enough. The results of this study are expected to provide important information from the viewpoints of cost and manufacturing process.

\section{CONCLUSION}

Based on the results obtained from the FTIRM measurements, we revealed that when ceramide-containing
$\operatorname{acResin}^{\circledR}$ adhesive sheets are attached to cultured skin sheets for several days, the ceramide molecules are released from the adhesive sheet. This study also showed that FTIRM spectroscopy is a powerful and suitable method to determine the local concentration of ceramide molecules in materials that do not contain molecules with amide bonds. In this study, however, we could not obtain experimental evidence that objectively indicates that the released ceramide molecules penetrate the cultured skin. Hence, one of the remaining tasks is to develop a method to ascertain whether the ceramide in the stratum corneum is intrinsic or exogenous.

Finally, judging from the present results and the preliminary results for the human skin, we would like to insist that the human skin barrier function-improving effects of the model medical sheets reported by Numata et $a l .{ }^{13)}$ are due to the ceramide molecules released from the adhesive materials containing ceramide.

\section{ACKNOWLEDGMENT}

We would like to thank Dr. N. Ohta(SPring-8) for his advice to recommend FTIRM measurements as the best suitable method for the aim of this study. FTIRM measurements were performed at beamline BL43IR of SPring-8 with the approval of the Japan Synchrotron Radiation Research Institute (JASRI) (Proposal No. 2015B1581, 2016A1511 and 2016B1577).

\section{DISCLOSURE}

HT was supported by funds from Alcare Corporation (Tokyo, Japan) regarding research on the structure of ceramide in adhesive materials. RW and NK are an employee of Alcare Corporation. The authors have no other conflicts of interest to disclose related to this work.

\section{References}

1) Duarte, A.P.; Coelho, J.F.; Bordado, J.C.; Cidade, M.T.; Gil, M.H. Surgical adhesives: Systematic review of the main types and development forecast. Prog. Polym. Sci. 37, 1031-1050 (2012).

2) Karp, J.M.; Langer, R.; Materials science: Dry solution to a sticky problem. Nature 477, 42-43 (2011).

3) Laulicht, B.; Langer, R.; Karp, J.M.; Quick-release medical tape. Proc. Natl. Acad. Sci. USA 109, 18803$18808(2012)$

4) Lund, C. Medical adhesives in the NICU. Newborn Infant Nurs. Rev. 14, 160-165(2014).

5) Konya, C.; Sanada, H.; Sugama, J.; Okuwa, M.; Ka- 
matani, Y.; Nakagami, G.; Sakaki, K. Skin injuries caused by medical adhesive tape in older people and associated factors. J. Clin. Nurs. 19, 1236-1242 (2010).

6) Bouwstra, J.A.; Ponec, M.; The skin barrier in healthy and diseased state. Biochim. Biophys. Acta 1758, 2080-2095 (2006).

7) Wertz, P.W. Skin ceramides in Lipids and Skin Health (Pappas, A. ed.). Springer, New York, pp. 67-74 (2015).

8) Norlén, L. Skin barrier, structure, and properties. in Lipids and Skin Health (Pappas, A. ed.). Springer, New York, pp. 25-42 (2015).

9) Melnik, B.; Hollman, J.; Plewig, G. Decreased stratum corneum ceramides in atopic individuals-a pathobiochemical factor in xerosis? Br. J. Dermatol. 119, 547549 (1988).

10) Imokawa, G.; Abe, A.; Jin, K.; Higaki, Y.; Kawashima, M.; Hidano, A. Decreased level of ceramides in stratum corneum of atopic dermatitis: an etiologic factor in atopic dry skin? J. Invest. Dermatol. 94, 89-96 (1994).

11) Meckfesse, M.H.; Brandt, S. The structure, function, and importance of ceramides in skin and their use as therapeutic agents in skin-care products. J. Am. Acad. Dermatol. 71, 177-184(2014).

12) Jin, K.; Higaki, Y.; Takagi, Y.; Higuchi, K.; Yada, Y.; Kawashima, M.; Imokawa, G. Analysis of beta-glucocerebrosidase and ceramidase activities in atopic and aged dry skin. Acta Derm. Venereol. 74, 337-340 (1994).

13) Numata, S.; Kambara, N.; Iwasaki, T.; Effect on barrier function improvement of peristomal skin by skin barrier contined ceramide. J. Jpn. Soc. Stoma \& Continence Rehabilitation 23, 9-18(2007). (in Japanese).

14) Jenquin, M.R.; Liebowitz, S.M.; Sarabia, R.E.; McGinity, J.W. Physical and chemical factors influencing the release of drugs from acrylic resin films. J. Pharm. Sci. 79, 811-816 (1990).

15) Takahashi, H.; Takada, K.; Nishimura, K.; Watanabe, R. Thermal-history-dependent phase behavior of ce- ramide molecular assembly in a UV-curable acrylic adhesive resin. J. Oleo Sci. 67, 905-915 (2018).

16) Schumacher, K.-H.; Sanborn, T. UV-curable acrylic hot melts for PSAs. in Adhesives and Sealants Industry Magazine June/July (2001). (www.adhesivesmag. com).

17) Scherzer, T.; Tauber, A.; Mehnert, R. UV curing of pressure sensitive adhesives studied by real-time FTIR-ATR spectroscopy. Vibr. Spectrosc. 29, 125-131 (2002).

18) Takahashi, H.; Takada, K.; Obata, Y.; Shimizu, N.; Ishida. K. Structure and phase behavior of synthetic ceramide $2((2 S, 3 R)$-2-octadecanoylamino-octadecane-1,3-diol) and its interaction with limonene. Trans. Mat. Res. Soc. Japan 40, 73-76 (2015).

19) Ishida, K.; Obta, Y.; Akagi, C.; Onuki, Y.; Takayama, K. Practical syntheses of D-erythro- and L-threo-ceramide[NDS] and difference in contribution of each isomer in microstructure of stratum corneum intercellular lipid. J. Drug Del. Sci. Tech. 24, 689-693 (2014).

20) Tinois, E; Tiollier, J.; Gaucherand, M.; Dumas, H.; Tardy, M.; Thivolet, J.; In vitro and post-transplantation differentiation of human keratinocytes grown on the human type IV collagen film of a bilayered dermal substitute, Exp. Cell. Res. 193, 310-319(1991).

21) Netzlaff, F.; Lehr, C.-M.; Wertz, P.W.; Schaefer, U.F. The human epidermis models EpiSkin ${ }^{\circledast}$, SkinEthic $^{\circledast}$ and EpiDerm ${ }^{\oplus}$ : An evaluation of morphology and their suitability for testing phototoxicity, irritancy, corrosivity, and substance transport. Eur. J. Pharm. Biopharm. 60, 167-178(2005).

22) Ikemoto, Y.; Moriwaki. T.; Hirono, T.; Kimura, S.; Shinoda. K.; Matsunami. M.; Nagai. N.; Nanba, T.; Kobayashi, K.; Kimura, H. Infrared microspectroscopy station at BL43IR of SPring-8. Infrared Phys. Tecnol. 45, 369-373 (2004).

23) Moriwaki, T.; Ikemoto, Y. BL43IR at SPring-8 redirected. Infrared Phys. Tecnol. 51, 400-403 (2008). 